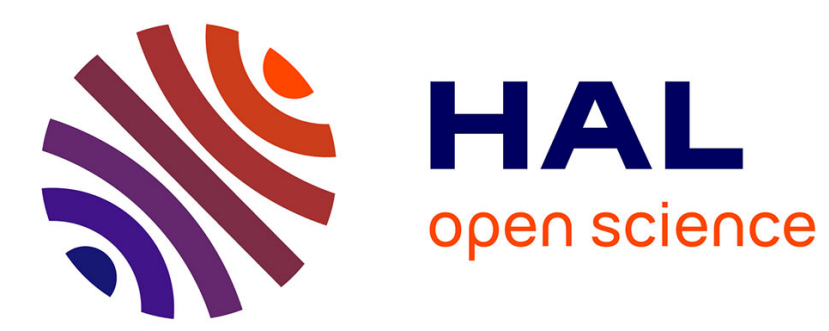

\title{
Miroirs interférentiels X et X-UV. Un cas particulier de multicouches métalliques à faibles périodes P. Dhez
}

\section{To cite this version:}

P. Dhez. Miroirs interférentiels $\mathrm{X}$ et $\mathrm{X}-\mathrm{UV}$. Un cas particulier de multicouches métalliques à faibles périodes. Journal de Physique IV Proceedings, 1996, 06 (C7), pp.C7-109-C7-115. 10.1051/jp4:1996712 . jpa-00254500

\section{HAL Id: jpa-00254500 https://hal.science/jpa-00254500}

Submitted on 1 Jan 1996

HAL is a multi-disciplinary open access archive for the deposit and dissemination of scientific research documents, whether they are published or not. The documents may come from teaching and research institutions in France or abroad, or from public or private research centers.
L'archive ouverte pluridisciplinaire HAL, est destinée au dépôt et à la diffusion de documents scientifiques de niveau recherche, publiés ou non, émanant des établissements d'enseignement et de recherche français ou étrangers, des laboratoires publics ou privés. 


\title{
Miroirs interférentiels $X$ et $X-U V$. Un cas particulier de multicouches métalliques à faibles périodes
}

\author{
P. Dhez
}

Laboratoire de Spectroscopie Atomique et Ionique, et LURE, Université Paris Sud, 91405 Orsay cedex, France

\begin{abstract}
Résumé : Depuis les années 70 on sait préparer des miroirs interférentiels X et X-UV, analogues dans leur principe aux multícouches diélectriques employées dans le domaine visible. Pour cela, à partir de deux matériaux choisis, on doit déposer de façon reproductible et périodique un grand nombre de bicouches. Les interfaces doivent être abruptes et les épaisseurs de matériaux de quelques couches atomiques. Comme pour les autres types de multicouches, les principales méthodes de dépôt ont été essayées; celles par bombardement ionique apparaissent comme les mieux adaptées pour la préparation de ce type de structures. Peu de méthodes de contrôle in situ existent, bien qu'il reste souhaitable de déposer les couches en vérifiant continûment leurs caractéristiques. Pour ces miroirs, les principales méthodes de caractérisations sont basées sur la diffraction $\mathrm{X}$, si possible à la longueur d'onde et à l'angle d'incidence envisagés pour l'utilisation. La qualité des interfaces est déterminante pour les performances de réflectivité.
\end{abstract}

\section{INTRODUCTION ET HISTORIQUE.}

Les matériaux artificiellement structurés à l'échelle quasi atomique sont préparés et étudiés pour les nouvelles propriétés qu'ils peuvent présenter par rapport aux matériaux massifs. Actuellement ce sont surtout les propriétés liées au magnétisme qui semblent attirer l'attention. Les miroirs interférentiels pour le domaine X-UV et les neutrons lents ne sont qu'un cas particulier de multicouches; les matériaux employés étant sélectionnés pour la valeur de leurs indices aux énergies à réfléchir.

La motivation la plus ancienne pour maîtriser la préparation de multicouches d'épaisseur quasiatomique semble avoir été le souhait de les utiliser comme miroirs $\mathrm{X}$, en complément des cristaux naturels. Par dépôt alterné et répétitif de deux matériaux différents, on peut moduler la densité électronique dans la direction perpendiculaire à la surface. On se libère ainsi des limitations imposées à la fois par la composition des cristaux naturels et par leurs très faibles périodes. Pour une multicouche, la diffraction n'est notable que dans la direction symétrique à l'angle d'incidence. La formule approchée de Bragg classique $2 d . \operatorname{Sin} \Theta=N \lambda$ s'applique; $d$ est la période des couches déposées et $2 d$ la plus grande longueur d'onde que l'on peut réfléchir. Le libre choix de la période permet d'employer les multicouches comme monochromateur à très basse énergie, en desssous du keV, là où les cristaux naturels ne fonctionnent plus.

La première tentative de réalisation date des années 1923 [1] et 1930 [2]; la préparation de $\mathrm{Cd} / \mathrm{Ag}$ et $\mathrm{Au} / \mathrm{Ag}$ par dépôt électrolytique fut un échec. Un premier résultat positif fut obtenu une dizaine d'années plus tard par Dumond et Youtz avec $\mathrm{Au} / \mathrm{Cu}$ préparé par évaporation thermique[3, 4]. Étant donné la vitesse d'interdiffusion entre ces deux métaux, la structuration organisée avec une période de $10 \mathrm{~nm}$ disparut au bout de quelques semaines; mais une nouvelle méthode de mesure de vitesse d'interdiffusion entre métaux était trouvée.

La fabrication de multicouches diélectriques pour le visible est analogue du point de vue technologique. La maîtrise nécessaire fut acquise dès les années 50. Les matériaux diélectriques déposés sont plus stables et les périodes nécessaires plus importantes (environ $100 \mathrm{~nm}$ ). Par rapport aux multicouches à faible période envisagées précédemment, les interfaces peuvent être moins abruptes et donc présenter une rugosité plus importante. De plus, une méthode de contrôle in situ en lumière visible fut très tôt développée pour ajuster les épaisseurs successives des matériaux. 
Dans les années 60 , la préparation de multicouches métalliques fut reprise pour des périodes de 3 à $5 \mathrm{~nm}$ sur $\mathrm{Pb} / \mathrm{Mg}, \mathrm{Au} / \mathrm{Mg}$ et $\mathrm{Fe} / \mathrm{Mg}$, toujours par évaporation thermique [5]. Dans ce cas, la durée de vie la plus longue, estimée à 6 mois, fut obtenue avec le dernier couple de matériaux.

En 1972, E. Spiller chez IBM [6] relança l'intérêt pour les multicouches de très faible période contenant des métaux. Son but était de préparer des miroirs X-UV, afin de réaliser des optiques interférentielles sous incidence normale. Il fut le premier à indiquer les limites théorique de tels miroirs X-UV dans lesquels l'absorption est notable, à en démontrer la réalisation par bombardement électronique avec $\mathrm{MgF}_{2} / \mathrm{Au}$ [7] et à développer le premier réflectomètre X-UV in situ [8]. Les avantages de la préparation par bombardement ionique pour les très faible périodes et le grand nombre de couches nécessaires ont été démontrés dès 1978 [9].

La réalisation et l'étude de tels miroirs fut une réelle avancée pour les optiques X-UV. Par rapport aux autres types de multicouches, cela incita à explorer de nouveaux couples de matériaux tels que W/C, W/Si, et plus tard $\mathrm{Mo} / \mathrm{Si}$. Pendant cette période, la diffraction des rayons $\mathrm{X}$ par de tels milieux fut étudiée très soigneusement et à des longueurs d'ondes X-UV inhabituelles pour la diffraction X classique. Un autre point, important pour la caractérisation de ces milieux, fut l'effort de comparaison entre le formalisme habituellement employé en diffraction $X$ et celui précédemment développé indépendamment pour les miroirs interférentiels du visible. Grâce à ces études on a maintenant un point de vue unifié de l'optique depuis le visible jusqu'au $\mathrm{X}$ et, indépendamment de la longueur d'onde considérée, on peut interpréter les observations avec l'un ou l'autre des formalismes. E. Spiller [10] et T. Barbee [11]ont récemment résumés l'état présent des connaissances des miroirs entre visible et $\mathrm{X}$.

\section{MATÉRIAUX INTÉRESSANTS POUR L'OPTIQUE X-UV.}

Pour chaque domaine d'application les critères de choix des constituants d'une multicouche sont différents. En ce qui concerne les miroirs interférentiels X-UV, les règles sont simples et faciles à comprendre.

Rappelons que seuls des miroirs interférentiels composés de matériaux parfaitement transparents permettent d'obtenir une réflectivité de $100 \%$. En effet toute absorption correspond à de l'énergie dissipée dans les couches; celle ci ne peut donc pas se retrouver dans le rayonnement réfléchi. Comme les diélectriques sont très transparents pour le visible, les multicouches diélectriques y sont très efficaces. Par contre dans l'UV tous les matériaux deviennent absorbants, d'où la difficulté d'y étendre leur emploi. Cette remarque concernant l'absorption est aussi valable en dehors des ondes électromagnétiques. Par exemple, à longueur d'onde équivalente, des miroirs interférentiels pour neutrons pourront être plus efficaces que ceux pour rayons $\mathrm{X}$, car ces particules sont très faiblement absorbées. En présence d'absorption on devra rechercher la meilleure réflectivité par interface, de façon à faire travailler le minimum de périodes en profondeur. Ceci sera au détriment de la résolution spectrale qui, comme dans tout système interférentiel, augmente avec le nombre d'éléments participant à l'interférence constructive.

Pour une énergie X-UV donnée, les critères pour sélectionner les matériaux d'un miroir interférentiel se résument comme suit :

a) Rechercher le couple de matériaux donnant la meilleure réflectivité à leur interface.

b) Si plusieurs couples sont équivalents, choisir celui conduisant à l'absorption la plus faible par période.

c) Optimiser l'épaisseur relative des deux matériaux en fonction des indices.

Ceci suppose de connaître les indices aux énergies désirées. Il faut aussi disposer d'un programme de calcul de réflectivité tenant compte des parties réelle et imaginaire de l'indice, qui traduisent respectivement la réfraction et l'absorption.

Pour le domaine X-UV, une recherche systématique des meilleurs matériaux a été réalisée en 1988 [12] à l'aide des premières tables d'indices existantes. Elle a permis de comprendre dans quels domaines d'énergies certaines applications seraient plus aisées. Pour des raisons pratiques, tous les matériaux ne conviennent pas (point de fusion ou d'évaporation, radioactivité, toxicité, ....). Certains matériaux initialement écartés, comme le béryllium, ont ensuite été réintroduit. La figure 1 est un exemple de remise à jour récente pour le domaine de 2 à $15 \mathrm{~nm}$ [13]. 

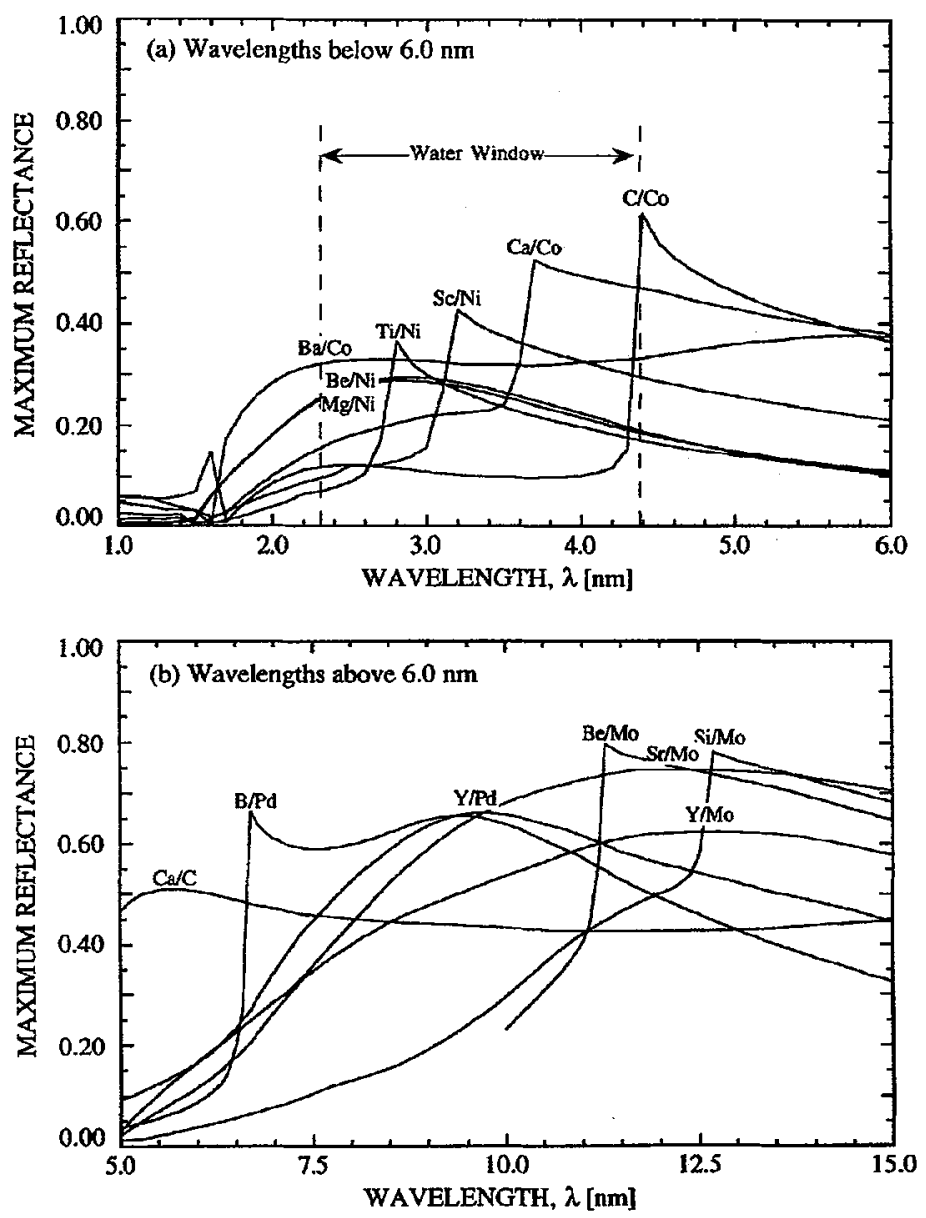

Figure 1 : Maximum de réflectivité théorique sous incidence normale, dans le domaine de 1 à $15 \mathrm{~nm}$, pour une multicouche parfaite de 500 périodes [12]. On remarquera la nécessité de changer de matériaux suivant le domaine d'énergie et la brusque variation de réflectivité lorsqu'on passe un seuil atomique de l'un des constituants.

Dans l'exemple ci-dessus, les réflectivités calculées supposent que la densité des matériaux déposés est celle du solide massif, les interface parfaites et les périodes absolument régulières. Comme pour toutes les multicouches, la modélisation et l'étude des interfaces restent l'objet d'un đébat. Les écarts à la notion d'interface parfaite sont le plus souvent globalement prises en compte par l'intermédiaire d'un facteur dit de Debye-Waller. Ce paramètre simple, bien adapté aux calculs, a initialement été proposé en diffraction $\mathrm{X}$ pour traduire l'effet de l'agitation thermique des atomes dans les cristaux. Il ne correspond évidemment à aucune réalité physique des interfaces. Actuellement, on tente de mieux rendre compte des observations expérimentales à l'aide de distributions de corrélations latérales et entre couches. Les mesures de diffusion hors spéculaires sont développées pour tester les nouveaux modèles à plusieurs paramètres. On trouvera ailleurs [10] une discussion sur ce point important pour tous les tests par diffraction $\mathrm{X}$. 


\section{QUELQUES ASPECTS PRATIQUES DE LA RÉLLISATION D'OPTIQUES X-UV.}

Ayant choisi un couple de matériaux adapté au domaine X-UV, il reste la difficulté commune à toutes les multicouches : réaliser une périodicité donnée avec une interface ayant une extension la plus faible possible. Cette extension est à comparer à l'épaisseur de la couche la plus mince du matériau déposé dans la période. La réflectivité $X$ est un des moyens pour vérifier la qualité des interfaces entre deux matériaux. En particulier on peut préparer une série de multicouches de période constante, mais en décroissant l'épaisseur de l'un des matériaux constituants. Pour une longueur d'onde choisie, en suivant la variation du rapport entre la réflectivité théorique et la réflectivité expérimentale, on peut évaluer l'épaisseur pour laquelle la couche n'est plus continue lorsque ce rapport change rapidement. En fait on observe pendant très longtemps des pics de Bragg, indiquant que la densité électronique est bien modulée parallèlement à la surface de la multicouches. Le minimum de modulation de densité électronique acceptable est fonction de l'application envisagée. A l'heure actuelle, dans les cas les plus favorables, on considère qu'au dessous d'environ $2 \mathrm{~nm}$ de période la perte de réflectivité due à l'imperfection des interfaces devient trop importante pour la plupart des applications en optique X-UV. En pratique, pour ce domaine, le rapport des épaisseurs entre les deux matériaux est de l'ordre de $1 / 3$, le matériau le plus absorbant devant avoir l'épaisseur la plus faible. Pour le domaine d'énergie de 0,1 à $4 \mathrm{keV}$, la figure 2 montre le diagramme de Dumond d'une multicouche W/C [14] typique des miroirs X-UV.

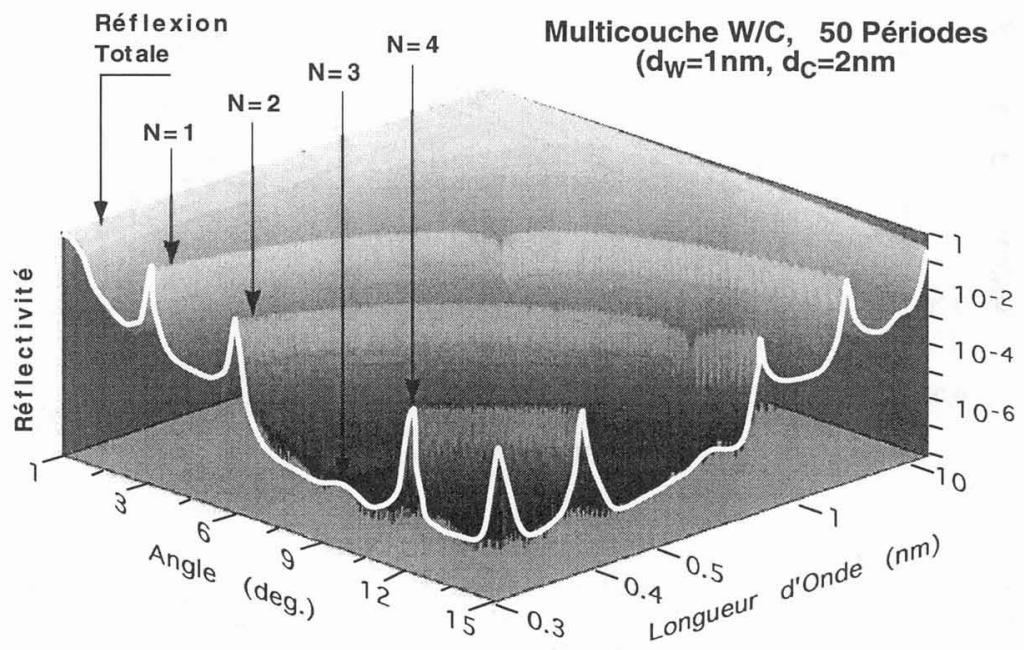

Figure 2 : Exemple de variation de réflectivité attendue dans le domaine $X-U V, 1-0,3 \mathrm{~nm}$ soit $0,1-4 \mathrm{keV}$, pour le couple W/C souvent employé [14]. Le rapport d'épaisseur des deux matériaux, optimisant habituellement la réflectivité dans ce domaine, conduit à une atténuation importante du 3ème ordre.

Pour les miroirs interférentiels, le contrôle in situ est le moyen le plus direct de vérifier et d'améliorer les conditions de dépôt : régularité des épaisseurs, densité des matériaux, rugosités, ...... . La plupart des méthodes mise au point pour les dépôts sous ultravide de couches épitaxiées par jet moléculaire ne sont pas utilisables en présence de sources d'électrons ou d'ions. L'ellipsometrie visible est l'une des rares méthodes convenables [15,16], mais l'interprétation des résultats nécessite un certain nombre d'hypothèses. Par ailleurs, pour les nombreuses couches nécessaires en X-UV, elle ne sonde que les dernières périodes déposées. Le test in situ par réflectivité $\mathrm{X}$ reste donc la solution la plus directe. Le système mis au point en 1980 par E. Spiller [8], fonctionne à longueur d'onde et angle fixes. Dans la relation de Bragg, les deux paramètres étant choisis, ceci détermine la période que l'on pourra contrôler. A titre d'exemple la figure 3, montre, comment après dépôt d'une légère surépaisseur déposée à dessein, l'usinage ionique sous contrôle de la réflectivité $\mathrm{X}$ permet de diminuer la rugosité obtenue lors du dépôt direct [17]. 


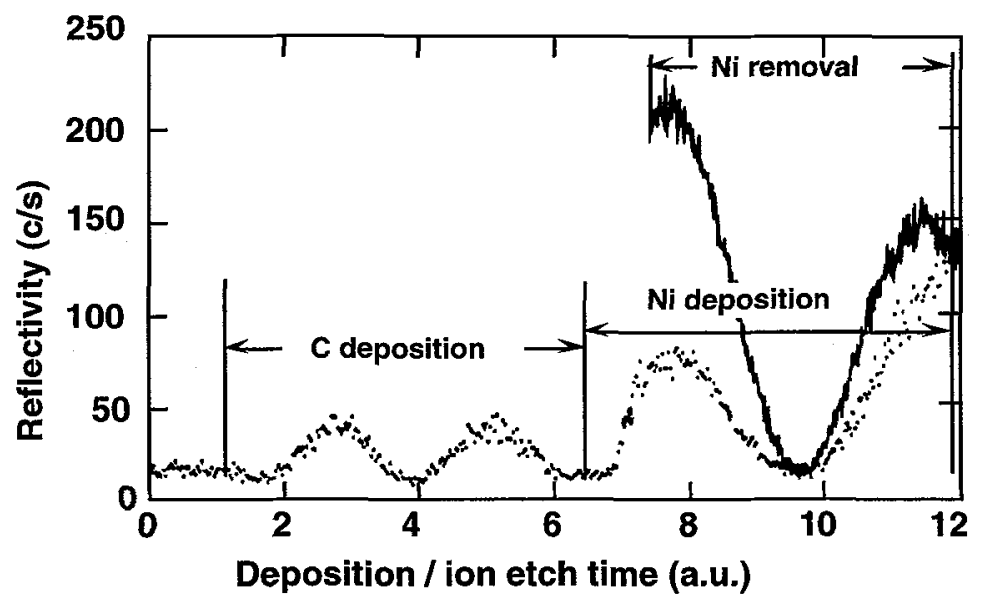

Figure 3 : Courbes expérimentales de réflectivité X-UV enregistrées in-situ, à longueur d'onde et angle fixes, pendant le dépôt d'un couche de carbone puis de nickel [17]. On voit que la périodicité de la variation de réflectivité permet de contrôler avec précision les épaisseurs déposées. Dans cet exemple, la couche de nickel à été bombardée après dépôt pour réoptimiser son épaisseur. Le gain en réflectivité obtenu, après usinage ionique, montre aussi la possibilité de diminuer la rugosité de la dernière interface métal-vide. Dans ce cas, la réflectivité est plus de deux fois supérieure à celle obtenue lors du dépôt de la couche initiale. Il est donc possible d'améliorer sous contrôle la qualité de chaque couche déposée.

En se reportant à la figure 2 on voit qu'on peut obtenir une information plus complète en observant simultanément plusieurs pics de Bragg. Ceci est possible en mesurant la réflectivité à longueur d'onde fixe à plusieurs angles, ou la réflectivité à incidence fixe à plusieurs longueur d'ondes. Ces deux méthođes respectivement appelées "dispersion angulaire" et "dispersion d'énergie" sont habituellement employées sur des échantillons après dépôt. Par contre, leur utilisation in situ est récente.

Un nouveau type de réflectomètre in situ, par dispersion angulaire avec un faisceau X monochromatique divergent, a récemment permis de suivre en parallèle la variation de réflectivité pour une certaine gamme d'angles d'incidences [14]. Le faisceau X monochromatique divergent est obtenu avec un cristal courbe et un tube X. Ainsi, sans déplacer aucun élément, on peut suivre simultanément la variation des pics de Bragg et donc plusieurs paramètres pendant toute la durée du dépôt. En cours de dépôt, on vérifie la période moyenne par l'écart entre les pics de Bragg correspondants aux différents ordres. Les largeurs de pics renseignent principalement sur la régularité des périodes déposées, leurs hauteurs relatives sur le rapport d'épaisseur des deux matériaux.

Un second type de réflectomètre in-situ, mais par dispersion d'énergie, vient de démontrer des possibilités analogues. Il utilise un faisceau polychromatique parallèle d'un tube $X$ sous incidence fixe[18]. Avec un détecteur $\mathrm{Si}(\mathrm{Li})$ fixe en position, l'analyse spectrale des photons réfléchis est possible car la bande passante de la multicouche est plus large que la résolution en énergie du détecteur choisi [19]. Dans ce cas, les différents pics de Bragg observés simultanément sont les harmoniques du premier ordre.

\section{EXEMPLES D'OPTIQUES X-UV.}

Dans le domaine X-UV il existe peu d'optiques hormis les miroirs sous réflexion totale; les multicouches ont donc peu à peu apporté des possibilités analogues à celles courantes dans le domaine visible. En dehors des optiques X-UV spécialisées pour l'imagerie, dont nous allons donner ensuite quelques exemples, on doit indiquer que les multicouches remplacent actuellement les cristaux organiques dans la majorité des récents appareils d'analyse par fluorescence $\mathrm{X}$, en particulier pour l'analyse des éléments légers. 
Une autre application commerciale récente est l'utilisation d'optiques courbes pour réfléchir sélectivement une raie de tube $X$, tout en focalisant ou en rendant parallèle le faisceau monochromatisé [20]. La figure 4 montre le principe de ces optiques qui tirent partie de la possibilité de déposer les multicouches sur des supports courbes, ou courbables dynamiquement, et avec un gradient de périodes contrôlé. Le contrôle đu gradient de période est important pour toutes les optiques courbes; il permet d'ajuster localement la période en fonction de l'angle d'incidence pour satisfaire en chaque point la loi de Bragg et ainsi conserver fixe la longueur d'onde réfléchie. Ce type de correction est employée aussi bien sous incidence rasante, dans les microscopes de type Kirkpatrick-Baez vers $0,1 \mathrm{~nm}$ [21], que dans les optiques sous incidence normale telles que les télescopes de type Ritchey-Chretien vers $1 \mathrm{~nm}$ [22].

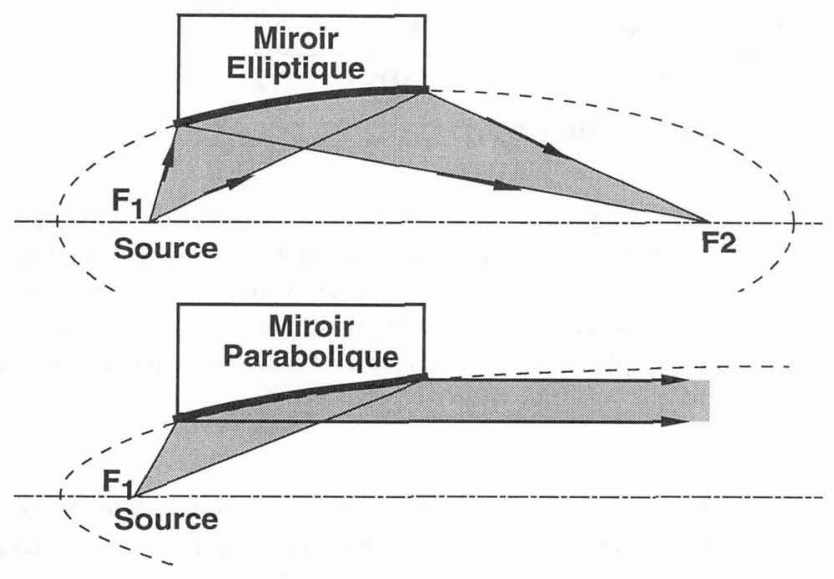

Figure 4 : Exemple d'optiques $\mathrm{X}$ multicouches à gradient de périodes commercialisées pour ,à la fois, monochromatiser et focaliser ou rendre parallèle le rayonnement des tubes à rayons X. La période de la multicouche est adaptée localement pour satisfaire en chaque point à la loi de Bragg en fonction de l'incidence.

La possibilité d'obtenir pour l'X-UV des polariseurs ou des analyseurs de polarisation a été démontrée en 1985 [23]. Pour l'angle de Brewster, $45^{\circ}$ dans ce domaine, il suffit d'optimiser la réflexion à la longueur d'onde choisie. La réalisation de multicouches semi-transparentes auto supportées permet de préparer des séparateurs de faisceaux ainsi que des polariseurs circulaires X-UV [24] et des interféromètres de type Mach-Zender pour le domaine X-UV [25].

L'usinage ionique de motifs submicroniques dans des multicouches ouvre la voie à des applications dépassant certainement les optiques X-UV. Comme pour les multicouches, le test par réflectivité constitue une bonne vérification de la précision d'usinage atteinte. En X et X-UV plusieurs applications de cette technologie sont en cours, l'une d'elle étant la préparation d'optiques dites de Bragg-Fresnel. Ce nouveau principe combine la diffraction de Fresnel de réseaux avec la réflectivité de Bragg d'un milieu périodique dans lequel est gravé le réseau. Les possibilités sont alors très variées, depuis le réseau linéaire strictement périodique [26] jusqu'à l'hologramme plan permettant la focalisation [27]. A titre d'exemple mentionnons la réalisation d'une microsonde $\mathrm{X}$ à $1 \mathrm{~nm}$ adaptée aux sources synchrotron [28].

Pour terminer, on peut faire état d'une autre retombée de la technologie des multicouches. A épaisseur égale déposée, la croissance de multicouches permet d'obtenir une surface moins rugueuse qu'une couche de matériau unique. Employée pour asphériser des miroirs de télescope, cette méthode est en cours de développement au NIST [29] pour corriger très localement l'épaisseur à l'aide de masques et ainsi préparer des surfaces ultra planes, ou des surfaces courbes non usinables avec précision par polissage à partir de surfaces sphériques. 


\section{CONCLUSION.}

Depuis les années 70 la maîtrise de préparation de milieux multicouches est activement poursuivie afin d'obtenir des propriétés physiques non disponibles avec les matériaux naturels massifs. Pour les miroirs interférentiels X-UV on s'intéresse à des couples de matériaux présentant un fort contraste d'indice aux énergies d'utilisation. Le plus souvent l'un des éléments est un métal, et les deux constituants ont des numéros atomiques très différents, ce qui écarte pratiquement toute possibilité d'épitaxie. Étant donné leur principe, les miroirs interférentiels ont des périodes comparables à la longueur d'onde à réfléchir, donc généralement inférieures à $10 \mathrm{~nm}$ pour 1 XX-UV, et on cherche à obtenir des interfaces abruptes. La réflectivité de ces miroirs étant étudiée à différentes longueurs d'onde ceci contribue à une meilleure connaissance des qualités des systèmes périodiques préparés et aux progrès des méthodes de dépôt des couches ultraminces. La mise au point de deux nouvelles méthodes de contrôle de dépôt in-situ pour les miroirs X-UV, l'une par dispersion angulaire et l'autre par dispersion d'énergie, devraient pouvoir aider à la préparation des autres types de multicouches.

\section{Références}

[1] Koeppe. H., Dissertation Gießen (1923).

[2] Deubner. W., Ann. der Physk. 5 (1930) 261-280.

[3] DuMond. J.W.M. et Youtz. J.P., Phys. Rev. 48 (1935) 703-???.

[4] DuMond. J.W.M. et Youtz., J.P. J. Appl. Phys. 11 (1940) 357-365.

[5] Dinklage. J., J. Appl. Phys. 38 (1967) 3781-3785.

[6] Spiller. E., Appl. Phys. Lett. 20 (1972) 365-367.

[7] Spiller. E., Appl. Opt. 15 (1976) 2333-2338.

[8] Spiller. E. et al., Appl. Phys. Lett. 37 (1980) 1048-1050.

[9] Barbee. T.W.and Keith. D.L., "Synthetic structures layered on the atomic scale" in Worshop on X-Ray Instrumentation for Synchrotron radiation research, Winick. H. and Brown. G., Eds. Standford Synchrotron Radiation Laboratory Report 7804 (1978) p. III-26,

[10] Spiller. E., "Soft X-Ray Optics", SPIE Optical Engineering Press, USA (1994).

[11] Barbee. T.W., "Synthesis of Multilayers", Symposium U2.5, MRS Spring Meeting, April 8-12, 1996, San Francisco (USA).

[12] Rosenbluth. A.E., Revue Phys. Appl. 23 (1988) 1599-1621.

[13] Montcalm. C et al., Appl. Opt., (à paraitre) 1996.

[14] Courbes aimablement calculée par T. Moreno avec son logiciel "X-Ray Vision".

[15] Ziegler. E. Communication à ce Colloque.

[16] Houdy. Ph., Revue Phys. Appl. 23 (1988) 1553-1659.

[17] Puik. E.J., Thésis University of Utrecht (Netherlands) 1990.

[18] J. C. Malaurent, H. Duval, G. Cauchon, P. Dhez, O. Hainaut, M, Idir and J.P. Chauvineau, Proceeding of 3rd Meeting Physics of Multilayers, Breckenbridge (Colorado) USA .(1996).

[19] Dhez.P, Duval. J.C. Malaurent, J. of X-Ray Sci. and Techn. 3, 176-193 (1992).

[20] Grupido. N, Gutman. G., "A general description of the Ovonyx Multilayers manufacturaed by Osmic Inc." 1788 Nortwood Drive, Troy, MI 48084 (USA).

[21] Underwood J.H., Thompson A.C., Kortright J.B. and Chapman K.C., Rev. Sci. Instr. in press (1996).

[22] Chauvineau. J.P. et al., SPIE 1546 (1991) 576-585.

[23] Khandar. A., P. Dhez.P., SPIE 563, 158-163 (1985).

[24] Kortright. J.B., Underwood. J.H., NIM A291, (1990) 272-277

[25] Wan. A.S., Da Silva. L.B.,Barbee. T.W. et al. SPIE 2520 (1995) 268-277 et 288-294.

[26] Kratsev. K., et al. NIM A368 (1996) 533-542.

[27] Idir. M, Mirone. A, Soullié. G, Dhez. P, Guerin. P.H, Ladan. F,Opt. Com. 119 (1995) 633-642

[28] Chevallier. P. et al. NIM A354, (1995) 584-587.

[29] Tarrio. C, Spiller. E, Evans. C.J, Lucatorto. T.B and Clark. C.W, "Post-polish figuring of optical surfaces using multilayer deposition" in Trends in Optics and Photonics, Vol. 4, pp. 144-148, Ed. by G.D. Kubiak and D.R. Kania, OPtical Society of America, Washington DC (1996). 